\title{
Proceeding
}

6th INSHS International Christmas Sport Scientific Conference, 11-14 December 2011. International Network of Sport and

Health Science. Szombathely, Hungary

\section{Didactics, movement and technology: new frontiers of the human-machine interaction}

\author{
STEFANO DI TORE ${ }^{1}$, FRANCESCA D'ELIA ${ }^{1}$, PAOLA AIELLO ${ }^{1}$, NADIA CARLOMAGNO², MAURIZIO \\ SIBILIO \\ ${ }^{1}$ Department of Human, Philosophical and Educational Sciences, University of Salerno, Italy \\ 2University of Suor Orsola Benincasa, Italy
}

\begin{abstract}
Di Tore S, D'Elia F, Aiello P, Carlomagno N, Sibilio M. Didactics, movement and technology: new frontiers of the human-machine interaction. J. Hum. Sport Exerc. Vol. 7, No. Proc1, pp. S178-S184, 2012. The current focus on the use of new technologies and media for teaching-learning purposes has led to an intensifying interest in the properties and peculiarities of educational videogames. Creating a learning environment within a video game might be an opportunity to capitalize and use in a constructive way the time that more and more teens spend playing video games. In light of this, it is particularly interesting the continuous evolution of computer videogames known as "exergames", a term derived from the joining of the words "exercise" and "games". Exergames are video games which encourage the emotional involvement and the sense of presence through interactions based on devices that allow a greater involvement of the body respect to typical controllers such as joysticks, joy pads, keyboards and mousses. These games involve the player in some form of exercise and physical activity through video games and specific feedback and data collection devices. The aim of this study, which is currently under development, is therefore to design an active and engaging learning environment in order to assess whether it is suitable to stimulate and facilitate the learning processes, particularly focusing on the functions of coordination and sensorimotor integration, through the typical interaction of exergames and exploiting the properties and peculiarities of the Microsoft Kinect system as a device to acquire data on the body movements of the player. Key words: MOTOR ACTIVITIES, EXERGAME, KINECT, VIDEOGAMES.
\end{abstract}

Corresponding author. Department of Human, Philosophical and Educational Sciences of the University of Salerno, Italy. 6th INSHS International Christmas Sport Scientific Conference, 11-14 December 2011. International Network of Sport and Health Science. Szombathely, Hungary

JOURNAL OF HUMAN SPORT \& EXERCISE ISSN 1988-5202

(c) Faculty of Education. University of Alicante

doi:10.4100/jhse.2012.7.proc1.20

VOLUME 7|Proc1 | 2012 | S178 


\section{INTRODUCTION}

The current focus on the use of new technologies and media for teaching and learning purposes has led to an intensifying interest in the properties and peculiarities of educational videogames. These are now considered a powerful tool that can revolutionize the current teaching-learning methodologies. Many scholars agree that they are the future of education (Dempsey et al., 1997; Coshott, 2009). Playing interactive video games is indeed an extremely widespread activity in Italy, just like it is in Europe, in the USA, in China, Japan, etc. Such activity seems to be a growing purely youth phenomenon. The main property of interactive video games is their potential to improve a high degree of involvement and fun and a strong motivation. The current focus on these tools in the teaching-learning field is aimed at assessing how they can increase learning, self-consciousness and skills' development. Creating a learning environment within a video game might be an opportunity to capitalize and use in a constructive way the time that more and more teens spend playing video games. However, today the researches carried out in the teaching and learning field about the educational potential of video games is still low. Probably the reason why the scientific literature in this field is still poor can be traced both to the current limited number of games created specifically for teaching-learning and educational purposes and to the difficulty of creating involving educational video games (Mason, 2010). In light of this, it is particularly interesting the continuous evolution of computer video games known as "exergames", a term derived from the joining of the words "exercise" and "games". It should be pointed out that the concept of exergame is not limited to the simple combination of "exercise" and "game", if so, the definition should also include sports such as football, volleyball, etc. Exergamingis instead an activity that combines exercise with the video games, where the word "exercise" refers to any activity that increases heart rate through the muscular effort and the word "video game" refers to an activity performed through the use of an electronic interface with rules, goals and feedback and implemented on computers, video game consoles or other devices (Young, 2009). Exergames are video games which encourage the emotional and cognitive involvement through interactions based on devices that allow a greater involvement of the body respect to typical controllers such as joysticks, joy pads, keyboard and mouse. These games involve the player in some form of exercise and physical activity through video games and specific feedback and data collection devices. The aim of this study, which is currently under development, is therefore to design an active and engaging learning environment in order to assess whether it is suitable to stimulate and facilitate the learning processes, particularly focusing on the functions of coordination and sensorimotor integration, through the typical interaction of exergames and exploiting the properties and peculiarities of the Microsoft Kinect system as a device to acquire data on the body movement of the player.

\section{MATERIAL AND METHODS}

The methodology has required the following steps:

1. Analysis of the literature.

2. Identification of the theoretical framework.

3. Analysis of the input devices on the market.

4. Design and implementation of a virtual learning environment.

5. Identification of scientifically validated tests to assess how the virtual learning environment affects the learning and visual-motor coordination processes.

6. Selection of a group of students attending the fifth year of the Italian primary school. 
7. Giving out a test to the students of the group.

8. Implementing the software in the class of the selected group of students.

9. Giving out a test to the students of the group.

10. Survey of the results obtained.

11. Analysis of data.

The first and second phase of the methodology allowed us to identify the visual-motor integration as one of the main cognitive element which is suitable to be stimulated and supported by the use of an exergame. The visual-motor integration is one of the preconditions of the learning processes and as well as being particularly essential in the acquisition of the instrumental skills of reading and writing, it fosters higher levels of cognitive processes involved in school learning activities (Marsha \& Amundson, 1994). However, in formal educational settings, it is not always considered important enough to recognize its true value. Currently, the scientific literature seems to recognize that the movement plays a crucial role in the integration of different mental processes. In this regard, Hannaford has suggested that "Every time we move in an organized ... manner, full brain activation and integration occurs, and the door to learning opens" (Hannaford, 1995). In this perspective the visual-motor responses may be considered as the first sensory integration of the development and so they result as one of the key objectives that every educational system should pursue. Hence, no wonder that the development of our software has been aimed at facilitating and supporting this function through specific interaction modes. The purpose of the third phase has been to analyze the devices of the motion sensor associated with the currently in circulation exergames in order to identify the input device more suited to the development of our virtual learning environment. The attempt to create ways of interacting with a high level of body involvement within video games is a fairly old idea. The continuous evolution of this type of video games has indeed led over time to the development and distribution of controllers, mostly belonging to the "motion sensor" technology that can detect information about the movement of the subject. Among the first motion sensor devices there is the Sony Eye Toy, released in 2002; it is a new camera peripheral for the PlayStation 2 (PS2). It is able to capture gestures or more general human poses and movements with a high degree of approximation.

In 2006, Nintendo released the Nintendo Wii console. It is a new home video game console whose controllers have motion sensors. The Wii controller, known as the Nunchuk, contains a motion-sensing technology and it can be connected to the main controller, so making it a two-handed normal device. These systems, which allow you to interact with virtual scenarios, have valuable educational potentials, because of their high degree of cognitive immersion. The main flaw of these systems is that they detect the subject movement, but they do not identify the body segments that make it at that time. The Kinect system, originally developed by Microsoft as part of the playful technology, is exempt from the above limit, since it is able to detect and identify different body segments. This feature is definitely functional to the research in the educational field of motor activities. The ability to identify the movement of specific body segments allows the potential development of teaching methodologies aimed at supporting the learning processes through experiences achieved through the interaction based on the body involvement. Moreover, the cost and portability of this system would help its introduction in schools for preventive as well as teachinglearning purposes, with particular attention to primary schools. In light of the above, the use of Kinect to acquire data on the subjects' movements has appeared to us as a completely natural choice. Hence it seems appropriate a more detailed analysis of the main technical characteristics of this system by Microsoft. Kinect (Figure 1) is equipped with cameras that make use of infra-red (IR) illumination to obtain data about depth, colour images and sound. The IR is used as a distance ranging device. The system can measure distance with a $1 \mathrm{~cm}$ accuracy at $2 \mathrm{~m}$ and has a resolution of $3 \mathrm{~mm}$ at $2 \mathrm{~m}$. This system can be equipped with a body tracking software and can be connected with a normal pc. 


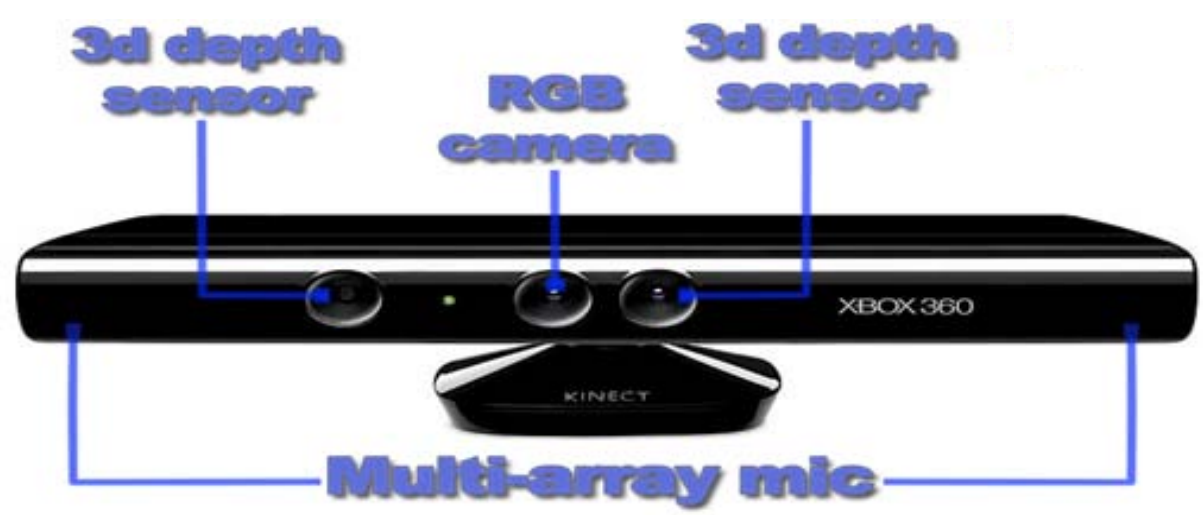

Figure 1. kinect equipation.

At this stage of development the Kinect system is able to identify 20 body segments (Figure 2).

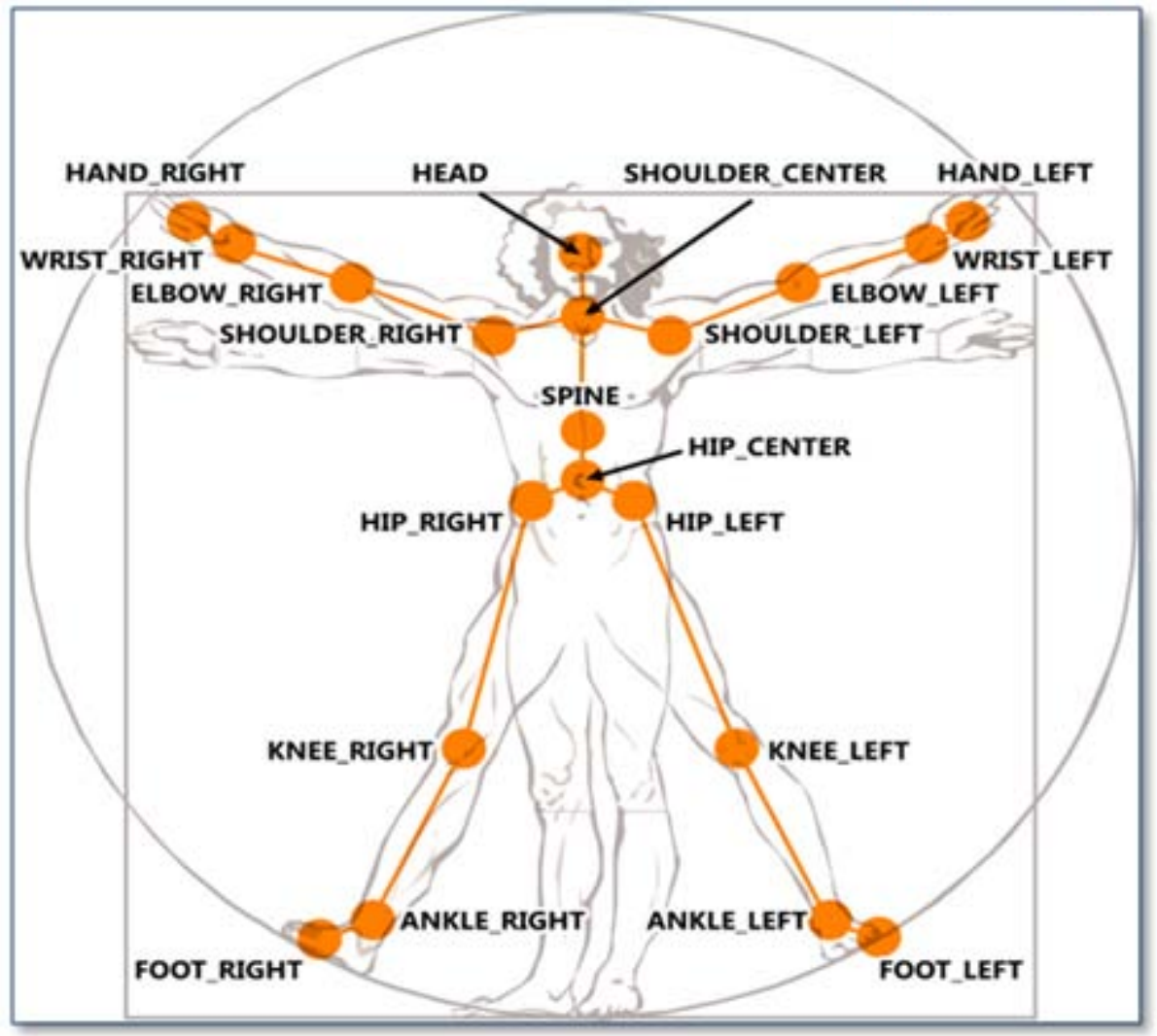

Figure 2. Kinect system body segments. 
Once identified the cognitive function to act upon and the data acquisition device to use, we proceeded to the realization of the fourth phase of the methodology described above that is to design and to carry out the first in a series of modules to be implemented within a virtual learning environment. The module achieved is a shape-game where the subject has to recognize shapes, rotate them and place them in special areas of the screen, as shown in the Figure 3.

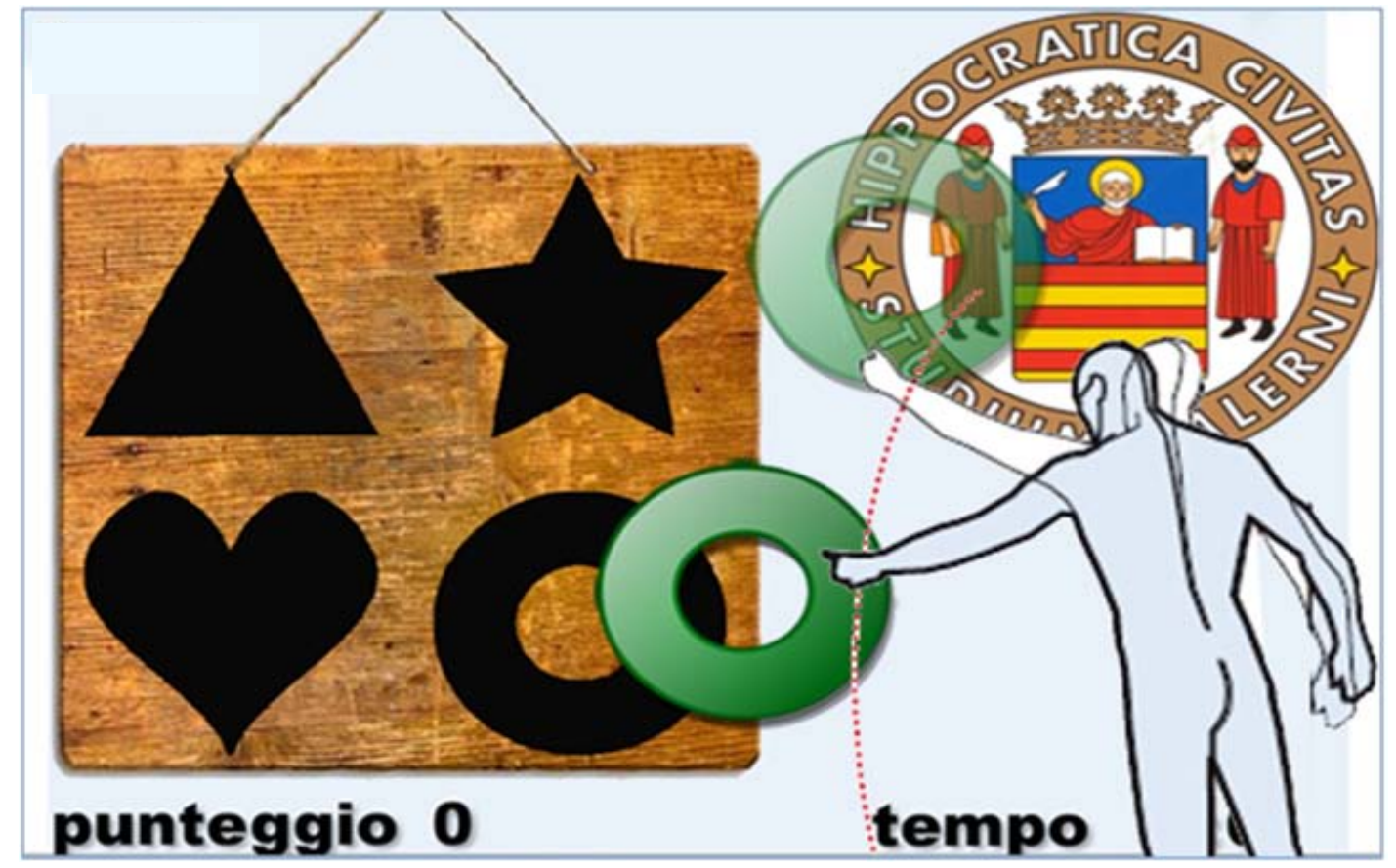

Figure 3. Shape-game.

The fifth phase of the methodology led to the identification of the VMI Test as an instrument ideal for measuring the progresses achieved through the use of the form produced. The VMI TEST (Visual-Motor Integration-test) is a "paper and pencil" test, scientifically validated, that requires the subject to copy a sequence of geometric shapes. The test was designed to assess the ways in which individuals integrate their visual and motor skills. The VMI Test is also a test virtually independent of the culture it belongs. For this reason, it is based on geometric shapes that go beyond the cultural aspects and not on numbers or letters that have a close relationship with the culture. In addition, the VMI test is one of the best and most accurate assessment tools for the integration of visual and motor skills. The module has been carried out in compliance with the theory of the VMI TEST. The shape-game shares the same shapes with the VMI and it aims at encouraging and stimulating the function of visual-motor integration measured by the test. The subsequent phases of the methodology have not been implemented yet. 


\section{DISCUSSION AND CONCLUSION}

If, after finishing the procedures of the described methodology, the results show that the form produced could effectively support and facilitate the learning processes and the functions of sensory-motor integration, it will be possible to achieve new teaching-learning methods, characterized by a strong cognitive and emotional involvement and aimed at stimulating learning processes through the use of the body and the technology.

\section{REFERENCES}

1. COSHOTT, R. Tests for Exergames. Retrieved 04/06/2009, from http://exergaming.pbwiki.com/Tests-for-Exergames

2. DEMPSEY, J.V. \& L. B. (1997). An Exploratory Study of Forty Computer Games. COE Technical Report No. 97-2

3. FADINI, B., \& SAVY, C. Informatica per le scienze umane. Milano: FrancoAngeli. 1999.

4. HANNAFORD, C. (1995). Smart moves. Arlington, VA: Great Ocean. 1995.

5. MARSHA, W. \& AMUNDSON, S. Relationship between visuomotor and handwriting skills of children in kindergarten. American Journal of Occupational Therapy. 1994; 48(11): 982-983.

6. MASON, J. T. A Research Proposal on Exergame Based Learning in an ESE Classroom, Retrieved 22/12/2011, from http://faculty.irsc.edu/FACULTY/JMason/Transmedia_Story_Project_Proposal_MASON.pdf

7. YOUNG, B. Exergaming Definitions. Retrieved 04 02, 2009, from http://www.gamesforhealth.org/index3.html 